\title{
GMR
}

\section{A microRNA-152 that targets the phosphatase and tensin homolog to inhibit low oxygen induced-apoptosis in human brain microvascular endothelial cells}

\author{
Y.H. Cao ${ }^{1 *}$, D.G. $\mathrm{Li}^{2 *}$, B. Xu${ }^{1}$, M.Q. Wang ${ }^{1}$, N. Zhen ${ }^{1}$, L.X. Man ${ }^{1}$, \\ Y.Y. Zhang ${ }^{1}$ and M. Chi ${ }^{1}$ \\ ${ }^{1}$ Department of Pediatrics, General Hospital of Military Command Jinan, \\ Jinan, Shandong, China \\ ${ }^{2}$ Department of Pediatric Surgery, \\ The Second Affiliated Hospital of Shandong University, Jinan, Shandong, China \\ *These authors contributed equally to this study. \\ Corresponding author: Y.H. Cao \\ E-mail: caoyanhuayelei@sina.com
}

Genet. Mol. Res. 15 (2): gmr.15027371

Received October 13, 2015

Accepted November 19, 2015

Published May 13, 2016

DOI http://dx.doi.org/10.4238/gmr.15027371

\begin{abstract}
Brain damage caused by perinatal asphyxia is dangerous for neonatal infants, but the mechanism by which it occurs remains elusive. In this study, microRNA-152 (miR-152) expression was induced by low oxygen levels in rat models of hypoxia brain damage, as well as in human brain microvascular endothelial cells (HBMECs) cultured in vitro. Analysis of the sequence of miR-152 revealed that the phosphatase and tensin homolog gene (PTEN) is probably the target of miR-152 both in humans and rats. When HBMECs were transfected with miR-152 mimics, PTEN expression was inhibited at both the mRNA and protein levels. Moreover, transfection with the miR-152 mimic also inhibited apoptosis induced by hypoxia. Furthermore, expression of the pro-apoptotic gene Bax was downregulated while the anti-apoptotic
\end{abstract}


gene $B c l 2$ was upregulated after miR-152 mimic transfection. Taken together, these results indicate that miR-152 induced by hypoxia suppresses cell apoptosis and acts as a protective factor during hypoxia by repressing PTEN.

Key words: miR-152; PTEN; Hypoxia; Apoptosis; Brain damage

\section{INTRODUCTION}

Hypoxic-ischemic brain disease (HIBD) in newborn infants is a damage to the brain caused by partial or total hypoxia, or decreased cerebral blood supply to the brain due to perinatal asphyxia (Shian et al., 1994). The consequences of HIBD for the infant are variable and depend on the severity of the hypoxia (Douglas-Escobar and Weiss, 2012; Chalak et al., 2014). While full recover is possible for some mild cases, sequelae of the central nervous system can also occur to different extents (Douglas-Escobar and Weiss, 2012; Chalak et al., 2014). During this process, hypoxia leads to a set of complicated pathological events including cell apoptosis (Zweckberger et al., 2006; Salmaso et al., 2014).

MicroRNAs (miRNAs) are short non-coding RNA molecules that negatively regulate gene expression through mRNA (Ambros, 2004). Because there may be multiple targets of a single miRNA, dysregulation of miRNA expression is thought to play an important role in the progression of a variety of diseases (Jansson and Lund, 2012). However, little attention has been paid to the role played by miRNA during infant brain damage caused by hypoxia. Recent reports have demonstrated that the level of certain miRNAs such as miR-30b and miR-182 is upregulated during damage to the brain caused by lack of oxygen, and these miRNAs are involved in the regulation of cellular apoptosis after injury (Ding et al., 2015; Han et al., 2015).

By utilizing a well-established hypoxia rat model of HIBD (Zhang et al., 2013), we investigated the molecular mechanism of brain damage due to hypoxia in neonates. During our miRNA assay screening, we occasionally found that miRNA-152 (miR-152) was significantly changed in the rat brain tissue as well as in human brain microvascular endothelial cells (HBMECs). Further analysis indicated that the phosphatase and tensin homolog gene (PTEN) could be the putative target of miR-152, which was further verified by our in vitro study. Moreover, overexpression of miR-152 could promote cell survival and inhibit apoptosis in vitro. In summary, these results will benefit the development of therapeutic strategies for treating infant brain damage caused by hypoxia.

\section{MATERIAL AND METHODS}

\section{Ethics statement}

The animal procedures used in this study were issued by the Ministry of Science and Technology (Beijing, China) and approved by the General Hospital of Jinan Command. All handling procedures were conducted according to recommendations proposed by the General Hospital of Jinan Command and all efforts were made to minimize suffering. Animals were housed in a temperature-controlled room with proper darkness-light cycles, and were fed a regular diet. 


\section{Rat model of low oxygen brain injury, tissue preparation, and miRNA microarray}

Six newborn male Sprague Dawley (SD) rats were purchased from the Chinese Academy of Military Science (Beijing, China). The rats $(\mathrm{N}=3)$ were exposed to low oxygen $\left(2 \% \mathrm{O}_{2}\right)$ for $24 \mathrm{~h}$ while the controls $(\mathrm{N}=3)$ remained in a normal environment. The frontal cortex was removed from the brains of the rats by micro-dissection, as previously described (Chiu et al., 2007) The RNAs were then purified with TRIzol ${ }^{\circledR}$ Reagent (Invitrogen, Carlsbad, CA, USA) according to manufacturer instructions. RNase-free DNase (Promega, Madison, WI, USA) was used to remove DNA from the RNA isolation procedure.

RNA samples were pooled from both groups and hybridized to the Agilent Rat miRNA Microarray Release 16.0, 8x15K (Agilent Technologies, Santa Clara, CA, USA) following the manufacturer protocol. Arrays were scanned using an Agilent Microarray Scanner and Feature Extractor (software v9.5.1). Real-time polymerase chain reaction (qPCR) was used to confirm the presence of miRNA-152 (hereafter referred to as miR-152) in the arrays.

\section{Cells, miR-152 mimics, and chemicals}

HEK293T cells maintained in Dulbecco's Modified Eagle's Medium (DMEM) supplemented with $10 \%$ fetal bovine serum (Gibco, Carlsbad, CA, USA) were used only for the reporter assay. The primary HBMECs (ACBRI 376) were commercially purchased from Cell System (Kirkland, WA, USA) and maintained in CSC Complete Medium (Cell System, 4Z0-500) according to the manufacturer instructions. Transfection of HEK293T or HBMEC with plasmid DNA or the miRNA mimic was conducted using Lipofectamine ${ }^{\mathrm{TM}}$ 2000 (Invitrogen, Grand Island, NY, USA) according to the manufacturer instructions. The miR-152 mimic MISSION ${ }^{\circledR}$ microRNA Mimic hsa-miR-152 and its scrambled control (miRcontrol) were commercially purchased from Sigma (Sigma-Aldrich, St. Louis, MO, USA).

\section{Reporter plasmid and luciferase-based miRNA functional assay}

The human PTEN 3'-untranslated region (UTR) reporter plasmid psiCHECK2PTEN-3'-UTR (Addgene plasmid \# 50936) was obtained from Addgene (Cambridge, MA, USA). The activity of luciferase in the HEV293T cells transfected with psiCHECK2-PTEN3'UTR along with miR-152 or the miR-control was evaluated using a Dual-Glo ${ }^{\circledR}$ Luciferase Assay System (Promega) according to the manufacturer instructions. The luminescence signal was measured with a VICTOR3 ${ }^{\mathrm{TM}}$ Multilabel Counter (Perkin-Elmer, Waltham, MA, USA). Relative percentages of luminescence intensity were calculated by comparison with the miRcontrol transfected cells.

\section{Reverse transcription and qPCR}

RNA isolation from in vitro-cultured cells was conducted using TRIzol (Invitrogen). Complementary DNA (cDNA) generation for the quantification of miRNA expression was carried out using a Hairpin-it ${ }^{\mathrm{TM}}$ miRNA RT-PCR Quantitation Kit (GenePharma, Shanghai, China) and ABM hsa-miR-152 Primers (Applied Biological Materials Inc., Richmond, BC, Canada) according to the manufacturer instructions. For cellular gene transcripts quantification, reverse transcription via AMV reverse transcriptase (Promega) was carried out using a 
combination of oligo dT and a random hexamer according to the manufacturer instructions, and qPCR detection with SYBR Green Mix (Life Technologies) for the targeting genes, as described previously (Patel et al., 2008; Patel et al., 2010). Transcripts of the glyceraldehyde 3-phosphate dehydrogenase gene $(G A P D H)$ and U6 small nuclear RNA (snRNA) were also amplified from the same sample to serve as an internal control for the cellar gene or miRNA normalization, respectively. Gene expression was quantified using the $2^{-\Delta \Delta \mathrm{Ct}}$ method (Livak and Schmittgen, 2001). The primers used in this study are listed in Table 1.

Table 1. Primers and their sequence.

\begin{tabular}{l|l}
\hline Primer name & Sequence (5' to 3') \\
\hline Has-miR-152 primers & N/A (purchased from ABM*) \\
\hline U6 F & CTCGCTTCGGCAGCACA \\
\hline U6 R & AACGCTTCACGAATTTGCGT \\
\hline PTEN F & TGTGGTCTGCCAGCTAAAGG \\
\hline PTEN R & CGGCTGAGGGAACTCAAAGT \\
\hline GAPDH F & CAGCCTCAAGATCATCAGCA \\
\hline GAPDH R & TGTGGTCATGAGTCCTTCCA \\
\hline
\end{tabular}

*Applied Biological Materials Inc., Richmond, BC, Canada.

\section{Flow cytometry-based cell apoptosis assay}

Cells were transfected with the miR-152 mimic or the scrambled control, and treated accordingly. The cells were then trypsinized, fixed with $70 \%$ ethanol, and permeabilized with phosphate-buffered saline containing 1\% TritonX100 (Sigma-Aldrich). A total of $1 \mathrm{x}$ $10^{6}$ HBMECs were stained with fluorescein isothiocyanate-labeled annexin $\mathrm{V}$ and propidium iodide. The stained cells were analyzed using a flow cytometry machine (FACSCalibur ${ }^{\mathrm{TM}}$, BD Biosciences, San Jose, CA, USA) for apoptosis analysis.

\section{Western blotting}

Sodium dodecyl sulfate polyacrylamide gel electrophoresis (SDS-PAGE) and western blotting were carried out as described previously (Patel et al., 2010; Nan et al., 2012). Briefly, after the denatured proteins had been transferred onto a polyvinylidene difluoride membrane, the membrane was blocked and probed by rabbit anti-Bax (Santa Cruz Biotechnology, Santa Cruz, CA, USA) and anti-Bcl-2 (Santa Cruz Biotechnology) antibodies. Specific reactions between the different antibodies and the corresponding proteins were detected using goat anti-rabbit antibody conjugated with horseradish peroxidase (Sigma-Aldrich, St. Louis, MO, USA), and were revealed by a chemiluminescence substrate. The antibody targeting GAPDH (Santa Cruz Biotechnology) was also included to normalize the total protein loading. The chemiluminescence signal was digitally recorded and analyzed using the ChemiDoc XRS imaging system (Bio-Rad, Hercules, CA, USA) with the Quantity One Program (version 4.6, Bio-Rad).

\section{Statistical analysis}

Statistical analysis was conducted using Excel (Microsoft) and graphs of the data were produced using Prism version 5.0 (GraphPad Software). Comparisons between the different groups were subjected to the Student $t$-test. A two-tailed P value of less than 0.05 was considered significant. 


\section{RESULTS}

\section{miR-152 expression significantly increased after hypoxic treatment in the SD rats}

During our microRNA array screening for the significantly changed microRNAs in the brain tissue after hypoxic treatment of SD rats, we noticed that miR-152 was one of the most upregulated microRNAs in response to hypoxic treatment (unpublished data). To confirm that these findings were applicable to humans, we used primary human brain microvascular endothelial cells (HBMECs) to verify the results observed in rats. The HBMECs were subjected to hypoxia $\left(2 \% \mathrm{O}_{2}\right)$ and total RNA was extracted at indicated time-points for validation of miR-152 expression by qPCR. The results indicated that the upregulation of miR-152 was time-dependent in the hypoxic group and increased $4 \mathrm{~h}$ after exposure. The expression of miR-152 reached a peak at $24 \mathrm{~h}$ (5.6-fold) and started to decline in the HBMECs $48 \mathrm{~h}$ after exposure (Figure 1A).

A

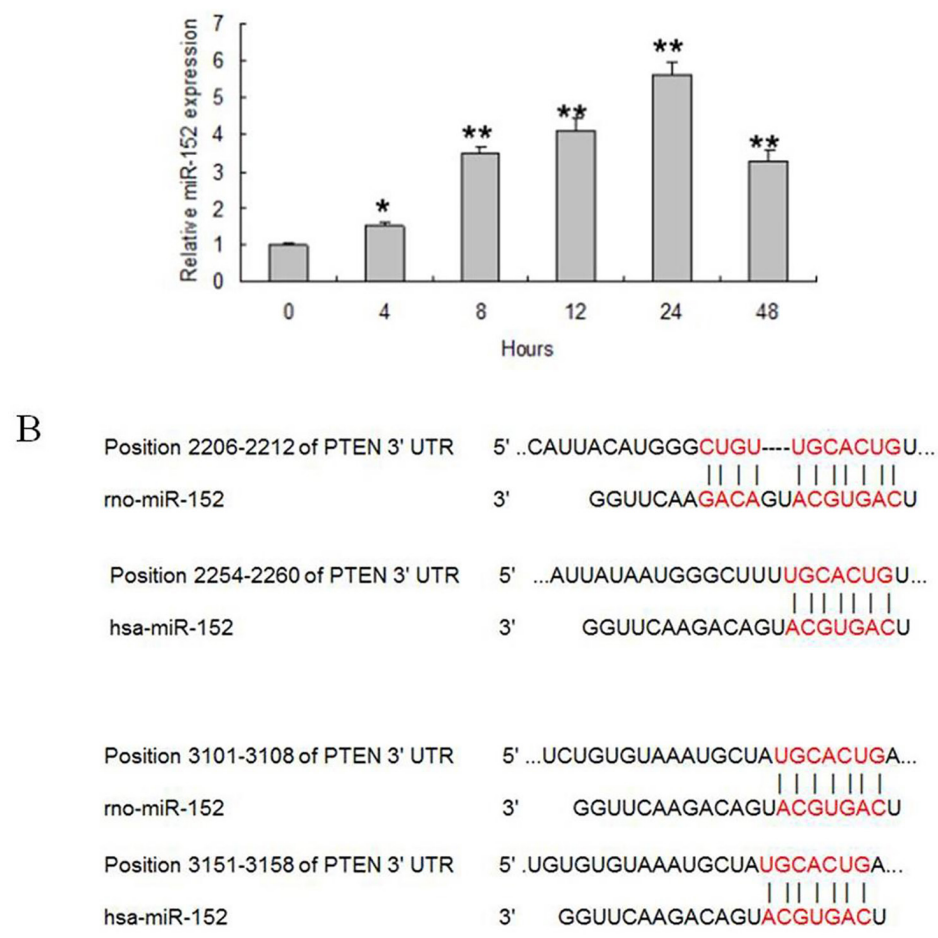

Figure 1. miR-152 expression was stimulated by hypoxic treatment and the phosphatase and tensin homolog gene $(P T E N)$ was predicted as the target for miR-152. A. miR-152 expression in human brain microvascular endothelial cells (HBMECs) subjected to hypoxia treatment. Cells were transfected with miR-152 for $48 \mathrm{~h}$, then subjected to low oxygen for the indicated time-points. Cells from each group were then harvested using TRIzol and subjected to realtime polymerase chain reaction (qPCR) detection of miR-152. B. miR-152 sequence alignment for the 3' untranslated region (UTR) of rat and human PTEN mRNA. Nucleotides paired between miR-152 and PTEN3'UTR human and rat sequences are shown in red. Significant differences are marked with asterisks $(* *)$, which means $\mathrm{P}<0.05$. 
Although, our data demonstrate that miR-152 can be induced by deprivation of oxygen, the role played by miR-152 during hypoxia treatment is still unclear. By analysis of the potential targeting sequence of rat miR-152 as well as its counterpart in humans, we noticed that the 3'UTR of the PTEN gene contains two putative binding sites for miR-152 with a slide position variation between rats and humans (Figure 1B). Owing to the action of its phosphatase protein product, PTEN is considered a tumor suppressor gene (Chu and Tarnawski, 2004). As PTEN is involved in the regulation of the cell cycle, preventing cells from over-proliferation, as well as cell apoptosis (Chu and Tarnawski, 2004; Hao et al., 2009) it is possible that hypoxia-induced miR-152 protects the cell by targeting PTEN at the mRNA level.

\section{PTEN is the target gene of miR-152}

To verify that $P T E N$ is the real target gene of miR-152, we first used a luciferase reporter vector containing a PTEN 3'-UTR to determine whether overexpression of miR-152 inhibited luciferase activity. In Figure 2A, compared with cells transfected with the miRcontrol, expression of luciferase was reduced to only 0.6-fold in the miR-152-transfected group, suggesting binding of miR-152 to the 3'-UTR of the PTEN mRNA. Moreover, besides the luciferase reporter assay, we also tested the level of endogenous PTEN mRNA by qPCR in the HBMECs with miR-152 or miR-control transfection. It was shown that transfection of miR-152 resulted in a 70\% reduction of PTEN mRNA compared with the control (Figure 2B). Furthermore, western blotting also revealed a significant reduction in the PTEN protein and confirmed the results of qPCR (Figure 2C).

\section{Transfection of miR-152 inhibits apoptosis induced by hypoxia in HBMECs}

Apoptosis can be induced in various cell types in response to hypoxia, and many proteins participate in this process (Chu and Tarnawski, 2004). As PTEN has been considered a tumor suppressor for many cancer cells and overexpression of PTEN leads to apoptosis (Hao et al., 2009), we expected that the inhibition of PTEN via miR-152 would inhibit apoptosis induced by hypoxia. When the miR-control and miR-152 were transfected into HBMECs and the cells were subjected to hypoxia $\left(2 \% \mathrm{O}_{2}\right)$, cell apoptosis was determined by flow cytometry. Our data revealed that there was little increase in the number of miR-control-transfected cells undergoing apoptosis compared with MOCK. However, after subjection to hypoxia, the number of cells undergoing early and late apoptosis increased dramatically to 22.9 and $3.82 \%$, respectively (Figure 3A). However, transfection with miR-152 inhibited the expression of PTEN, and cells experiencing early and late apoptosis were reduced to 9.52 and $2.02 \%$ (Figure $3 \mathrm{~A})$, respectively, suggesting that miR-152 plays a protective role in HBMECs during hypoxia.

Moreover, it was also interesting to note the expression of pro-apoptotic and antiapoptotic genes in HBMECs after PTEN expression had been inhibited by miR-152. The bestknown anti-apoptotic proteins, B-cell lymphoma 2 (Bcl-2) (Tsujimoto et al., 1984) and Bcl2-associated X protein (Bax), another member of the Bcl-2 family (Oltvai et al., 1993), were examined in this study. As shown in Figure 3B, hypoxia treatment of HBMECs did not change the expression of Bcl-2 but upregulated the pro-apoptotic Bax. However, with the transfection of miR-152, Bcl-2 expression was significantly increased. In contrast, without hypoxia stimulation, the Bax expression level was only 0.41 -fold compared with the cells transfected with the miR-control. A similar expression pattern was also observed in the hypoxia-treated 
cells (Figure 3B), suggesting that the downregulation of Bax may be one possible explanation for apoptosis inhibition in miR-152-transfected cells. Taken together, our data suggest that miR-152 protects against hypoxia-induced cell apoptosis by inhibiting PTEN expression.

A

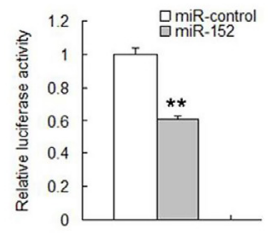

$\mathrm{B}$

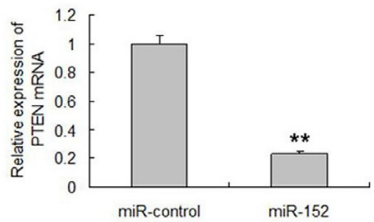

C

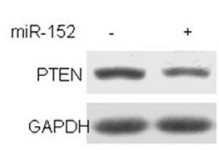

Figure 2. Phosphatase and tensin homolog gene $(P T E N)$ is the regulating target of miR-152. A. Reporter assay for miR-152 target in PTEN 3' untranslated region (UTR). HEK293 cells were first transfected with the PTEN 3'UTR reporter vector for $24 \mathrm{~h}$, then transfected with the miR-152 mimic and miR-control. After $48 \mathrm{~h}$, the luciferase activity in the cells was determined. B. PTEN mRNA expression in miR-152 mimic-transfected cells. Human brain microvascular endothelial cells (HBMECs) were transfected with the miR-152 mimic or miR-control for $48 \mathrm{~h}$, then the cells were harvested using TRIzol for real-time polymerase chain reaction (qPCR) analysis of the PTEN mRNA expression. C. Western blotting for PTEN protein following PTEN mRNA expression in the miR152 mimic-transfected cells. HBMECs were transfected with the miR-152 mimic or miR-controls for $48 \mathrm{~h}$, then the cells were harvested for sodium dodecyl sulfate polyacrylamide gel electrophoresis (SDS-PAGE) and western blotting. Significant differences are marked with asterisks $(* *)$, which means $\mathrm{P}<0.05$.

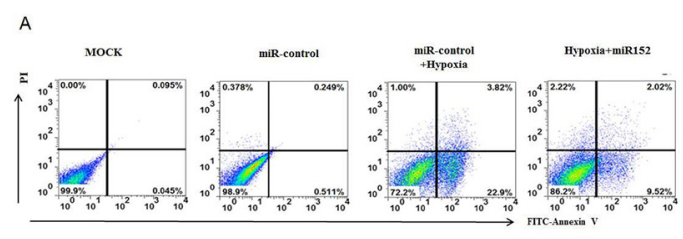

B

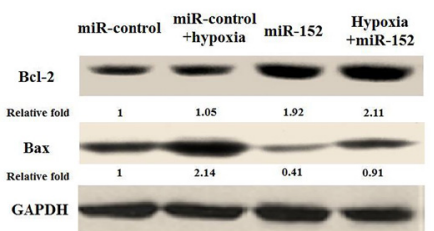

Figure 3. miR-152 inhibiting apoptosis by targeting the phosphatase and tensin homolog gene (PTEN). A. Flow cytometry-based apoptosis assay. Human brain microvascular endothelial cells (HBMECs) were transfected with miR-152 or the miR-control, and subjected to hypoxia treatment. The cells were fixed, stained, and examined for apoptosis by flow cytometry. B. Western blotting for expression of anti-apoptotic and pro-apoptotic genes. HBMECs were treated in the same way as for flow cytometry, then analyzed by sodium dodecyl sulfate polyacrylamide gel electrophoresis (SDS-PAGE) and western blotting for the indicated genes. 


\section{DISCUSSION}

miRNAs play an important role in gene expression regulation (Ambros, 2004). It has been demonstrated that miRNAs are involved in the regulation of many of the biological functions of vascular endothelial cells, including proliferation, migration, and differentiation (Ambros, 2004). Overexpression or downregulation of miRNAs can drive the protection or damage of vascular vessels (McDonald et al., 2012). In this study, miR-152 was identified as a protector antagonizing apoptosis in brain vascular endothelial cells through the direct targeting of PTEN.

miR-152 is thought to be a tumor-suppressing miRNA. The authors of one report have suggested that miR-152 acts as a tumor suppressor in prostate cancer by targeting the 3'UTR of TGF- $\alpha$ (Zhu et al., 2013). Other researchers reported epigenetic silencing by DNA hypermethylation of miR-152 in endometrial cancer, and restoration of miR-152 expression in endometrial cancer cell lines resulted in inhibition of tumor cell growth, both in vitro and in vivo (Tsuruta et al., 2011). However, in our study, we found that miR-152 could be induced by hypoxia and inhibited hypoxia-induced apoptosis in vascular endothelial cells. Moreover, miR-152 could target PTEN to protect against apoptosis.

$P T E N$ is one of the critical negative regulators of the PI3K-Akt signaling pathway and is considered a tumor suppressor gene (Chu and Tarnawski, 2004; Wang and Jiang, 2008). The cellular functions of PTEN include regulation of proliferation, cell growth, migration, genomic stability, and stem cell self-renewal (Wang and Jiang, 2008). PTEN activity can be regulated by mutations, epigenetic silencing, transcriptional repression, aberrant protein localization, and post-translational modifications (Wang and Jiang, 2008). Dysregulation of PTEN expression and activation has been reported in many cancers (Li et al., 1997; Chen et al., 2005). Several miRNAs, such as miR-19a (Dou et al., 2015), miR-21 (Qi et al., 2009), miR-26a (Huse et al., 2009), and miR-214 (Schwarzenbach et al., 2012) have been identified as regulators of PTEN expression. In these reports, overexpression of miRNAs targeting PTEN has been considered a contributing factor for tumor development (Huse et al., 2009). However, as our study indicated, miR-152 is also a potential regulator of PTEN; most previous reports have indicated that miR-152 acts as a tumor suppressor by targeting proteins other than PTEN (Cheng et al., 2014; He et al., 2015; Huang et al., 2015). Therefore, although our data suggest a protective function of miR-152 during hypoxiainduced apoptosis in primary microvascular endothelial cells, the miR-152-PTEN relationship and its effects on the development and progression of cancer requires further investigation.

However, although PTEN does act as a tumor suppressor by inhibiting cell proliferation, it has also been demonstrated that PTEN has potential as a therapeutic target for gene deletion or mutation in tissue regeneration, especially in neuron tissue (Zhou et al., 2003; Ji et al., 2006). Therefore, it is possible that $P T E N$ plays a special role in protection or regeneration in neuron or brain tissue, which may be related to our observation. In conclusion, miR-152 is a hypoxia-induced miRNA that is upregulated in hypoxia-treated infant rat brain tissues as well as in human microvascular endothelial cells cultured in vitro. miR-152 can antagonize hypoxia-induced apoptosis by downregulation of PTEN, suggesting that miR-152 may protect the brain during conditions of low oxygen or low blood flow.

\section{Conflicts of interest}

The authors declare no conflict of interest. 


\section{REFERENCES}

Ambros V (2004). The functions of animal microRNAs. Nature 431: 350-355.http://dx.doi.org/10.1038/nature02871

Chalak LF, Sánchez PJ, Adams-Huet B, Laptook AR, et al. (2014). Biomarkers for severity of neonatal hypoxic-ischemic encephalopathy and outcomes in newborns receiving hypothermia therapy. J. Pediatr. 164: 468-74.e1.http://dx.doi. org/10.1016/j.jpeds.2013.10.067

Chen Z, Trotman LC, Shaffer D, Lin HK, et al. (2005). Crucial role of p53-dependent cellular senescence in suppression of Pten-deficient tumorigenesis. Nature 436: 725-730.http://dx.doi.org/10.1038/nature03918

Cheng Z, Ma R, Tan W and Zhang L (2014). MiR-152 suppresses the proliferation and invasion of NSCLC cells by inhibiting FGF2. Exp. Mol. Med. 46: e112.http://dx.doi.org/10.1038/emm.2014.51

Chiu K, Lau WM, Lau HT, So KF, et al. (2007). Micro-dissection of rat brain for RNA or protein extraction from specific brain region. J. Vis. Exp. 2007: 269.

Chu EC and Tarnawski AS (2004). PTEN regulatory functions in tumor suppression and cell biology. Med. Sci. Monit. 10: RA235-RA241.

Ding X, Sun B, Huang J, Xu L, et al. (2015). The role of miR-182 in regulating pineal CLOCK expression after hypoxiaischemia brain injury in neonatal rats. Neurosci. Lett. 591: 75-80. http://dx.doi.org/10.1016/j.neulet.2015.02.026

Dou L, Meng X, Sui X, Wang S, et al. (2015). MiR-19a regulates PTEN expression to mediate glycogen synthesis in hepatocytes. Sci. Rep. 5: 11602.http://dx.doi.org/10.1038/srep11602

Douglas-Escobar M and Weiss MD (2012). Biomarkers of hypoxic-ischemic encephalopathy in newborns. Front. Neurol. 3: 144. http://dx.doi.org/10.3389/fneur.2012.00144

Han F, Huo Y, Huang CJ, Chen CL, et al. (2015). MicroRNA-30b promotes axon outgrowth of retinal ganglion cells by inhibiting Semaphorin3A expression. Brain Res. 1611: 65-73.http://dx.doi.org/10.1016/j.brainres.2015.03.014

Hao LS, Zhang XL, An JY, Yao DM, et al. (2009). Adenoviral transduction of PTEN induces apoptosis of cultured hepatic stellate cells. Chin. Med. J. (Engl.) 122: 2907-2911.

He J, Yu JJ, Xu Q, Wang L, et al. (2015). Downregulation of ATG14 by EGR1-MIR152 sensitizes ovarian cancer cells to cisplatin-induced apoptosis by inhibiting cyto-protective autophagy. Autophagy 11: 373-384. http://dx.doi.org/10.1 $\underline{080 / 15548627.2015 .1009781}$

Huang H, Hu M, Li P, Lu C, et al. (2015). Mir-152 inhibits cell proliferation and colony formation of CD133(+) liver cancer stem cells by targeting KIT. Tumour Biol. 36: 921-928. http://dx.doi.org/10.1007/s13277-014-2719-x

Huse JT, Brennan C, Hambardzumyan D, Wee B, et al. (2009). The PTEN-regulating microRNA miR-26a is amplified in high-grade glioma and facilitates gliomagenesis in vivo. Genes Dev. 23: 1327-1337. http://dx.doi.org/10.1101/ gad. 1777409

Jansson MD and Lund AH (2012). MicroRNA and cancer. Mol. Oncol. 6: 590-610. http://dx.doi.org/10.1016/j. molonc.2012.09.006

Ji SP, Zhang Y, Van Cleemput J, Jiang W, et al. (2006). Disruption of PTEN coupling with 5-HT2C receptors suppresses behavioral responses induced by drugs of abuse. Nat. Med. 12: 324-329. http://dx.doi.org/10.1038/nm1349

Li J, Yen C, Liaw D, Podsypanina K, et al. (1997). PTEN, a putative protein tyrosine phosphatase gene mutated in human brain, breast, and prostate cancer. Science 275: 1943-1947. http://dx.doi.org/10.1126/science.275.5308.1943

Livak KJ and Schmittgen TD (2001). Analysis of relative gene expression data using real-time quantitative PCR and the $\left.2^{(-\Delta \Delta \mathrm{C}(\mathrm{T})}\right)$ Method. Methods 25: 402-408. http://dx.doi.org/10.1006/meth.2001.1262

McDonald RA, Hata A, MacLean MR, Morrell NW, et al. (2012). MicroRNA and vascular remodelling in acute vascular injury and pulmonary vascular remodelling. Cardiovasc. Res. 93: 594-604. http://dx.doi.org/10.1093/cvr/cvr299

Nan Y, Wang R, Shen M, Faaberg KS, et al. (2012). Induction of type I interferons by a novel porcine reproductive and respiratory syndrome virus isolate. Virology 432: 261-270.http://dx.doi.org/10.1016/j.virol.2012.05.015

Oltvai ZN, Milliman CL and Korsmeyer SJ (1993). Bcl-2 heterodimerizes in vivo with a conserved homolog, Bax, that accelerates programmed cell death. Cell 74: 609-619. http://dx.doi.org/10.1016/0092-8674(93)90509-O

Patel D, Opriessnig T, Stein DA, Halbur PG, et al. (2008). Peptide-conjugated morpholino oligomers inhibit porcine reproductive and respiratory syndrome virus replication. Antiviral Res. 77: 95-107. http://dx.doi.org/10.1016/j. antiviral.2007.09.002

Patel D, Nan Y, Shen M, Ritthipichai K, et al. (2010). Porcine reproductive and respiratory syndrome virus inhibits type I interferon signaling by blocking STAT1/STAT2 nuclear translocation. J. Virol. 84: 11045-11055. http://dx.doi. org/10.1128/JVI.00655-10

Qi L, Bart J, Tan LP, Platteel I, et al. (2009). Expression of miR-21 and its targets (PTEN, PDCD4, TM1) in flat epithelial atypia of the breast in relation to ductal carcinoma in situ and invasive carcinoma. BMC Cancer 9: 163. http://dx.doi. org/10.1186/1471-2407-9-163 
Salmaso N, Jablonska B, Scafidi J, Vaccarino FM, et al. (2014). Neurobiology of premature brain injury. Nat. Neurosci. 17: 341-346.http://dx.doi.org/10.1038/nn.3604

Schwarzenbach H, Milde-Langosch K, Steinbach B, Müller V, et al. (2012). Diagnostic potential of PTEN-targeting miR-214 in the blood of breast cancer patients. Breast Cancer Res. Treat. 134: 933-941. http://dx.doi.org/10.1007/ $\underline{\text { s10549-012-1988-6 }}$

Shian WJ, Chi CS, Chen JW and Hsieh KS (1994). Hypoxic-ischemic effect on infants and children with cyanotic congenital heart disease: clinical assessment of neurological examination and brain magnetic resonance images. Zhonghua Yi Xue Za Zhi 53: 154-157.

Tsujimoto Y, Finger LR, Yunis J, Nowell PC, et al. (1984). Cloning of the chromosome breakpoint of neoplastic B cells with the t(14;18) chromosome translocation. Science 226: 1097-1099. http://dx.doi.org/10.1126/science.6093263

Tsuruta T, Kozaki K, Uesugi A, Furuta M, et al. (2011). miR-152 is a tumor suppressor microRNA that is silenced by DNA hypermethylation in endometrial cancer. Cancer Res. 71: 6450-6462. http://dx.doi.org/10.1158/0008-5472. CAN-11-0364

Wang X and Jiang X (2008). Post-translational regulation of PTEN. Oncogene 27: 5454-5463. http://dx.doi.org/10.1038/ onc.2008.242

Zhang Q, Ding Y, Yao Y, Yu Y, et al. (2013). Creating rat model for hypoxic brain damage in neonates by oxygen deprivation. PLoS One 8: e83589. http://dx.doi.org/10.1371/journal.pone.0083589

Zhou XP, Waite KA, Pilarski R, Hampel H, et al. (2003). Germline PTEN promoter mutations and deletions in Cowden/ Bannayan-Riley-Ruvalcaba syndrome result in aberrant PTEN protein and dysregulation of the phosphoinositol-3kinase/Akt pathway. Am. J. Hum. Genet. 73: 404-411.http://dx.doi.org/10.1086/377109

Zhu C, Li J, Ding Q, Cheng G, et al. (2013). miR-152 controls migration and invasive potential by targeting TGF $\alpha$ in prostate cancer cell lines. Prostate 73: 1082-1089. http://dx.doi.org/10.1002/pros.22656

Zweckberger K, Erös C, Zimmermann R, Kim SW, et al. (2006). Effect of early and delayed decompressive craniectomy on secondary brain damage after controlled cortical impact in mice. J. Neurotrauma 23: 1083-1093. http://dx.doi. org $/ 10.1089 /$ neu.2006.23.1083 